\title{
Development
}

\section{EEG Phase-Amplitude Coupling Strength and Phase Preference: Association with Age over the First Three Years after Birth}

\author{
Michael G. Mariscal, ${ }^{1, *}$ (DApril R. Levin,, ${ }^{1, *}$ Laurel J. Gabard-Durnam, ${ }^{2}$ Wanze Xie, ${ }^{2}$ \\ Helen Tager-Flusberg, ${ }^{3}$ and Charles A. Nelson ${ }^{2,4}$
}

https://doi.org/10.1523/ENEURO.0264-20.2021

${ }^{1}$ Department of Neurology, Boston Children's Hospital, Harvard Medical School, Boston, MA 02115, ${ }^{2}$ Division of Developmental Medicine, Boston Children's Hospital, Harvard Medical School, Boston, MA 02215, ${ }^{3}$ Department of Psychological and Brain Sciences, Boston University, Boston, MA 02215, and ${ }^{4}$ Harvard Graduate School of Education, Cambridge, MA 02138

\begin{abstract}
Phase-amplitude coupling (PAC), the coupling of the phase of slower electrophysiological oscillations with the amplitude of faster oscillations, is thought to facilitate dynamic integration of neural activity in the brain. Although the brain undergoes dramatic change and development during the first few years of life, how PAC changes through this developmental period has not been extensively studied. Here, we examined PAC through electroencephalography (EEG) data collected during an awake, eyes-open EEG collection paradigm in 98 children between the ages of three months and three years. We employed non-parametric clustering methods to identify areas of significant PAC across a range of frequency pairs and electrode locations, and examined how PAC strength and phase preference develops in these areas. We found that PAC, primarily between the $\alpha-\beta$ and $\gamma$ frequencies, was positively correlated with age from early infancy to early childhood $\left(p=2.035 \times 10^{-6}\right)$. Additionally, we found $\gamma$ over anterior electrodes coupled with the rising phase of the $\alpha$ - $\beta$ waveform, while $\gamma$ over posterior electrodes coupled with the falling phase of the $\alpha$ - $\beta$ waveform; this regionalized phase preference became more prominent with age. This opposing trend may reflect each region's specialization toward feedback or feedforward processing, respectively, suggesting opportunities for back translation in future studies.
\end{abstract}

Key words: connectivity; cross frequency; development; EEG; phase-amplitude coupling; phase preference

\section{Significance Statement}

The brain undergoes significant development during infancy and early childhood, enabling the emergence of higher-level cognition. Phase-amplitude coupling (PAC) is thought to support the integration of information within the brain. Our data suggest PAC increases from three months to three years of age. We additionally report the anterior and posterior electrodes show opposing forms of PAC; this regional phase preference also increases with age of electroencephalography (EEG) collection. These findings help set the stage for future analyses of PAC in young children with neurodevelopmental disorders, in which development of biomarkers during early life is a burgeoning field and measures of cross-frequency coupling may offer new promise.

Received June 17, 2020; accepted May 10, 2021; First published May 26, 2021.

The authors declare no competing financial interests.
Author contributions: A.R.L., L.J.G.-D., H.T.-F., and C.A.N. designed research; M.G.M. and A.R.L. performed research; A.R.L., L.J.G.-D., and W.X. contributed unpublished reagents/analytic tools; M.G.M. and A.R.L. analyzed data; M.G.M. and A.R.L. wrote the paper. 


\section{Introduction}

Dynamic integration of neural activity across various timescales is a critical component of healthy brain function. Oscillatory brain activity, created by the synchronous firing of large ensembles of neurons, can stem from a variety of underlying mechanisms and index a variety of cognitive processes (Kopell et al., 2014). In the field of neurodevelopment, measurement of oscillatory activity within specific frequency bands has thus been of longstanding interest (Saby and Marshall, 2012). Cross-frequency coupling, which describes the interaction between different oscillatory frequencies, has gained interest recently given its potential to integrate neural information in a manner that is functionally relevant to both typical and atypical brain function (Canolty and Knight, 2010).

One form of cross-frequency coupling is phase-amplitude coupling (PAC), in which the phase of the low-frequency activity modulates the amplitude of the highfrequency activity. Increasing evidence implicates PAC in a variety of important functional processes. PAC strength and location has been shown to shift with task demands (Voytek, 2010). Additionally, alterations in PAC have been found in a variety of brain disorders, including schizophrenia, autism, attention deficit hyperactivity disorder, and Parkinson's (Salimpour and Anderson, 2019), highlighting the importance of PAC for healthy brain functioning.

Moreover, underlying characteristics of PAC may reflect an area's functional configuration within neural networks. PAC phase max, or the phase of the low-frequency waveform corresponding to the largest amplitude of the highfrequency waveform, has been found to differ by cortical layer (Sotero et al., 2015), interneuron function (Port et al., 2019), depth of anesthesia (Soplata et al., 2017), brain area (Ninomiya et al., 2015), coupling frequencies, and task performance (Jones et al., 2020; Lega et al., 2016). This suggests that PAC phase preference (as measured on the scalp) could reflect an underlying area's dynamic functional configuration during the recording period. Although many measures of PAC are agnostic to phase, these findings suggest that not just if, but how $\gamma$ interacts with lower frequency waveforms may reflect a region's role in either the intake or modulation of information for neural processing.

This work was supported by the Child Neurology Foundation (A.R.L.), University of Tokyo International Research Center for Neurointelligence (L.G. D.), and National Institute on Deafness and Communication Disorders Grants R01-DC010290 (to H.T.-F. and C.A.N.) and R21 DC 08637 (to H.T.F.).

${ }^{*}$ M.G.M. and A.R.L. contributed equally to this work and should be considered co-first authors.

Acknowledgements: We thank all the laboratory members who contributed to this study and the participants and their families who make this research possible.

Correspondence should be addressed to April R. Levin at april.levin@ childrens.harvard.edu.

https://doi.org/10.1523/ENEURO.0264-20.2021

Copyright (C) 2021 Mariscal et al.

This is an open-access article distributed under the terms of the Creative Commons Attribution 4.0 International license, which permits unrestricted use, distribution and reproduction in any medium provided that the original work is properly attributed.
Numerous developmental changes in electroencephalography (EEG) power have been documented (Saby and Marshall, 2012). However, no studies have examined the development of PAC phase preference, and few have examined the development of PAC strength over early childhood. Of note, coupling between high $\delta(2-4 \mathrm{~Hz})$ and a $40-\mathrm{Hz}$ frequency following response during a $40-\mathrm{Hz}$ auditory click train has been found to increase from 8 to 16 years in the first principal component of the EEG data back-projected onto $\mathrm{FCz}$, and begins to decrease from 16 to 22 years (Cho et al., 2015). Still, few studies have focused on infancy and early childhood, a period when the brain is highly plastic and shows significant structural and functional developmental changes. Although PAC has been documented in infancy (Vanhatalo et al., 2005), only one study examined PAC development during infancy: PAC between slow wave $(0.7-2 \mathrm{~Hz})$ and $\beta(11.3-32 \mathrm{~Hz})$ oscillations across the scalp during sleep was found to decrease over the first two weeks after birth (Tokariev et al., 2016). These studies suggest that PAC can vary based on the age, frequency range, topography, state, and task being studied.

Thus, although the brain continues to demonstrate high plasticity and significant developmental changes from birth to early childhood (Gao et al., 2017), no studies have examined how PAC strength or phase preference develops in this age range. This is of particular importance because many neurodevelopmental disorders first manifest behaviorally during these early years. Development of predictive or risk-associated biomarkers that may allow for earlier treatment is a high priority in the field of neurodevelopmental disorders (Sahin et al., 2019; Wolff and Piven, 2020). While EEG power has been extensively studied for this purpose (Brito et al., 2019; GabardDurnam et al., 2019), PAC may offer particular promise given its potential mechanistic underpinnings (Canolty and Knight, 2010; Jensen et al., 2014; Hyafil et al., 2015; Helfrich et al., 2016). However, use of PAC for studying neurodevelopmental disorders first requires an understanding of how PAC changes across typical development. Here, we sought to address these gaps by characterizing, through both PAC strength and phase preference, how PAC develops between three months and three years of age.

\section{Materials and Methods}

\section{Participants}

The data for this study were drawn from a larger longitudinal study of neurocognitive development across the first three postnatal years of life. This study included later born infants ( $n=98 ; 53$ males and 45 females; Table 1) who had at least one typically developing older sibling. The study was conducted at Boston Children's Hospital/ Harvard Medical School and Boston University. All infants had a minimum gestational age of 36 weeks, no history of prenatal or postnatal medical or neurologic problems, no known genetic disorders (e.g., fragile $X$, tuberous sclerosis) and no family history of autism spectrum disorder or other neuropsychiatric conditions (based on parent 
Table 1: Participant demographics

\begin{tabular}{ll}
\hline & Participants $(n=98)$ \\
\hline Mex & $53(\mathrm{M}) 45(\mathrm{~F})$ \\
$\quad<4$-year degree & $13(13 \%)$ \\
4-year degree & $26(27 \%)$ \\
Graduate degree & $49(50 \%)$ \\
Did not answer & $10(10 \%)$ \\
Paternal education & \\
<4-year degree & $19(19 \%)$ \\
4-year degree & $34(35 \%)$ \\
Graduate degree & $34(35 \%)$ \\
Did not answer & $11(11 \%)$ \\
Race & \\
White or White & $82(84 \%)$ \\
Black or African American & $3(3 \%)$ \\
Asian & $2(2 \%)$ \\
American Indian or Alaskan Native & $0(0 \%)$ \\
Native Hawaiian or Pacific Islander & $0(0 \%)$ \\
More than one reported & $10(10 \%)$ \\
Not reported & $1(1 \%)$ \\
Ethnicity & \\
Not Hispanic or Latino & $93(94 \%)$ \\
Hispanic or Latino & $4(4 \%)$ \\
Not reported & $1(1 \%)$ \\
\hline
\end{tabular}

report). Institutional review board approval was obtained from both institutions (\#X06-08-0374).

\section{EEG acquisition/processing}

Among infants enrolled in the study, EEG data were collected sequentially at $3,6,9,12,18,24$, and 36 months of age, as previously described (Gabard-Durnam et al., 2019). However, not all participants completed an EEG recording at every time point; 17 participants had an EEG completed at only one time point, 10 participants had an EEG completed at two time points, 21 participants had three time points, 22 participants had four, 19 participants had five, eight participants had six, and only one participant had an EEG completed at all seven timepoints. Infants were seated on their caregiver's lap while a research assistant blew bubbles and/or presented toys to ensure the infant remained calm, without any time-locked stimulus or task. Continuous EEG was recorded for up to 5 min using either a 64-channel Geodesic Sensor Net System or 128-channel Hydrocel Geodesic Sensor Nets (Electrical Geodesics). Data were sampled at either 250 or $500 \mathrm{~Hz}$, and referenced at collection to a single vertex electrode $(\mathrm{Cz})$. Impedances were kept below $100 \mathrm{k} \Omega$ (within recommended guidelines for young children, given the high-input impedance capabilities of this system's amplifier).

Raw EEG files were exported from NetStation to MATLAB (versionR2017b, MathWorks) for preprocessing. Files were processed using the batch EEG automated processing platform (BEAPP; Levin et al., 2018; Fig. 1). Data were first filtered using a $1-100 \mathrm{~Hz}$ bandpass filter, which had a combined transition band width of $1 \mathrm{~Hz}$. Data sampled at $500 \mathrm{~Hz}$ were then downsampled to $250 \mathrm{~Hz}$ for consistency, and to constrain the signal decomposed by later independent components analysis (ICA)-based

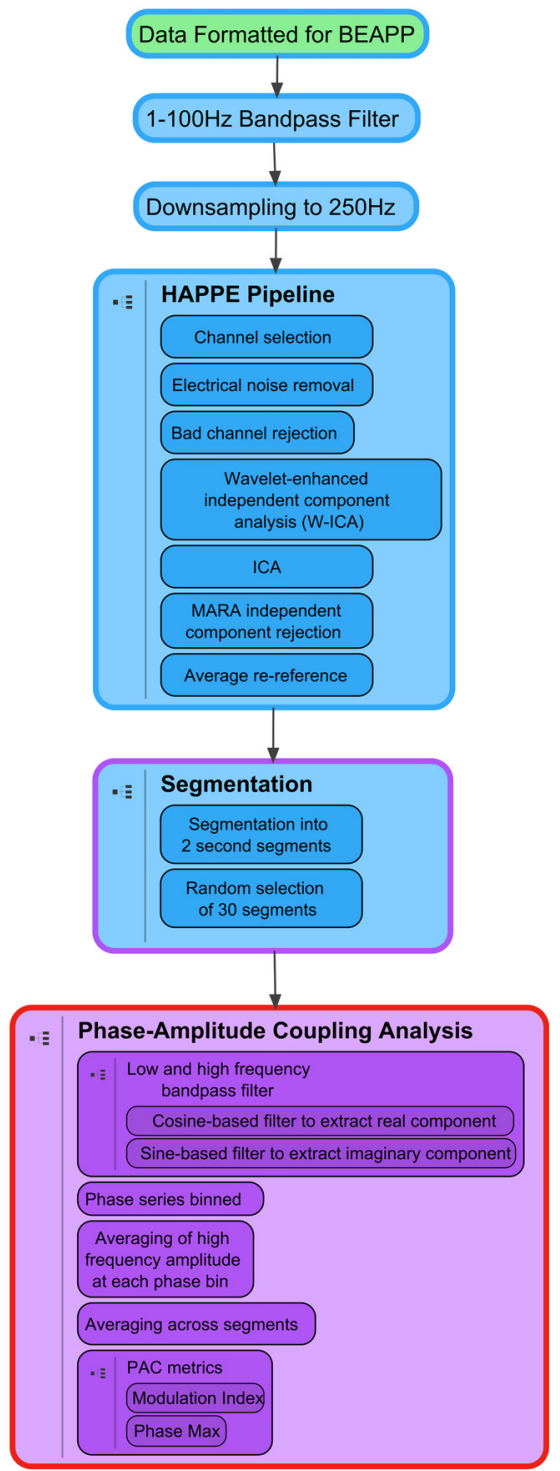

Figure 1. Processing pipeline: steps in EEG processing and PAC analysis. EEG data processing in BEAPP consists of several major steps: preprocessing (formatting, filtering, downsampling, HAPPE artifact rejection and re-referencing to average, segmentation) and PAC analysis; each step is represented by a cell, with additional details inside each cell. Cell body color reflects module input format, and cell outline color reflects module output format. Green = native file format. Blue = EEG in continuous array. Purple $=E E G$ in segmented $3 D$ array. Red $=$ PAC measures.

steps (Gabard-Durnam et al., 2018). Subsequently, within BEAPP, the Harvard Automated Preprocessing Pipeline for EEG (HAPPE), which was developed specifically to optimize preprocessing of developmental EEG data with potentially high levels of artifact and short recordings, was used to automate preprocessing and artifact minimization (Gabard-Durnam et al., 2018). Because ICA cannot reliably decompose data with a large number of channels given a short recording (Onton and Makeig, 2006; Gabard-Durnam et al., 2018), and because many other electrodes differed in their placements between the 64- 
Table 2: Number of EEG files (for each net type and in total) collected and analyzed per age group studied

\begin{tabular}{|c|c|c|c|c|c|c|}
\hline $\begin{array}{l}\text { Age } \\
\text { (months) }\end{array}$ & $\begin{array}{l}\text { 64-channel } \\
\text { geodesic } \\
\text { collected }\end{array}$ & $\begin{array}{l}\text { 64-channel } \\
\text { geodesic } \\
\text { analyzed }\end{array}$ & $\begin{array}{l}\text { 128-channel } \\
\text { HydroCel } \\
\text { collected }\end{array}$ & $\begin{array}{l}\text { 128-channel } \\
\text { HydroCel } \\
\text { analyzed }\end{array}$ & $\begin{array}{l}\text { Total } \\
\text { EEGs } \\
\text { collected } \\
\end{array}$ & $\begin{array}{l}\text { Total } \\
\text { EEGs } \\
\text { analyzed }\end{array}$ \\
\hline 3 & 5 & 2 & 13 & 12 & 18 & 14 \\
\hline 6 & 19 & 14 & 56 & 48 & 75 & 62 \\
\hline 9 & 21 & 14 & 65 & 54 & 86 & 68 \\
\hline 12 & 18 & 16 & 67 & 53 & 85 & 69 \\
\hline 18 & 9 & 6 & 56 & 46 & 65 & 52 \\
\hline 24 & 10 & 6 & 58 & 53 & 68 & 59 \\
\hline 36 & 0 & 0 & 76 & 62 & 76 & 62 \\
\hline
\end{tabular}

channel and 128-channel nets, only electrodes in the international 10-20 system were included in this pipeline; all other electrodes were removed before further analysis. In brief, within HAPPE, data underwent the following steps: electrical line noise removal at $60 \mathrm{~Hz}$ via Cleanline (Mullen, 2012), bad channel rejection (using channel power spectrum outlier thresholds), wavelet-thresholding of independent components, and secondary independent component analysis with component rejection via the automated multiple artifact rejection algorithm (MARA; Winkler et al., 2011, 2014). Data were then re-referenced to average, and segmented into 2-s windows for PAC analysis. This length was set to contain a sufficient sample of cycles for analysis of slower oscillations, while also retaining a sufficient amount of data (a longer segment length, in the presence of artifact, would lead to the rejection of more data). For each segment, to further remove artifact, the probability of an electrode's activity given the electrode's activity in all other segments, as well as the probability of an electrode's activity given the activity of all other electrodes in the same segment, was assessed using EEGLAB's pop_jointprob function (Delorme and Makeig, 2004). Segments where either probability was outside 3 SDs from the mean were rejected (GabardDurnam et al., 2018). Because $60 \mathrm{~s}$ of data has been shown to be needed to obtain stable PAC estimates (Berman et al., 2015), for each participant, 30 segments (60 s of data) were then randomly selected for further analysis; files with fewer than 30 segments of data at this stage were not analyzed (Table 2). Primary PAC metrics were then obtained as described below using code added to the BEAPP software, publicly available at https:// github.com/lcnbeapp/beapp.

Five EEG recordings were $>3$ SDs from the mean on one of the following HAPPE data quality output parameters: percent good channels, mean retained artifact probability, median retained artifact probability, percent of independent components rejected, and percent variance retained after artifact removal. These EEGs were evaluated for differences in overall PAC (averaged across frequencies and channels), all files were found to be within 2 SDs of the mean at the time point of collection, so these files were included in later analyses.

\section{Simulated signals}

Our EEG processing pipeline included multiple filtering steps. Given that filtering of EEG data has been shown to result in frequency dependent phase shifts (Yael et al.,
2018), we ran simulated signals through our pipeline to examine whether these filtering steps could alter observed PAC phase preference. To do so, a high-frequency oscillation was added on top of a low-frequency oscillation such that the high-frequency oscillation demonstrated increased amplitude during $-90^{\circ}, 0^{\circ}, 90^{\circ}$, and $180^{\circ}$ phases in four separate simulated signals. Simulated pink noise was then added to this signal to prevent artifact rejection from rejecting this unrealistic data. Because these phase shifts are frequency dependent, we generated these simulated phase-preferences in a variety of low-frequency and high-frequency combinations: specifically, between all combinations of the low-frequency oscillations of $2,4,8$, and $16 \mathrm{~Hz}$, and the high-frequency oscillation $64 \mathrm{~Hz}$. All simulated signals were run through all processing steps used in the present study with the exception of re-referencing, which removes the phase preference of this simulated data. After running PAC, the phase preference of each low-frequency phase and highfrequency amplitude combination was compared with its original phase preference.

\section{Computation of PAC metrics}

Modulation index

PAC was first quantified using the Pactools toolbox (Dupré la Tour et al., 2017) in Python as integrated within BEAPP. In detail, the signal in each segment was first exported from MATLAB into Python and filtered into a range of frequency pairs, with center frequencies for the low frequencies ranging from 2 to $20 \mathrm{~Hz}$ in $2-\mathrm{Hz}$ steps, and for the high frequencies ranging from $40-100 \mathrm{~Hz}$ in $4-\mathrm{Hz}$ steps. The low-frequency signal was filtered with a $2-\mathrm{Hz}$ bandwidth, and the high-frequency signal was filtered with a $20-\mathrm{Hz}$ bandwidth. Filtering at this step consisted of a zero-phase cosine-based filter to extract the real component, and then a sine-based filter to extract the imaginary component, resulting in a complex-valued output signal. Subsequently, PAC was assessed between each low-frequency and high-frequency filtered signal. Given the relatively wide bandwidth used when filtering the high frequencies, and to account for the transition bands of the filters, we did not analyze PAC frequency pairs in which there was overlap between the frequency response of the low-frequency filter and that of the high-frequency filter. For all other remaining low-frequency and high-frequency combinations, the time series of phases of the low-frequency signal and the amplitude of the high-frequency 
signal were obtained. The phases of the low-frequency signal were then binned into $1820^{\circ}$ intervals $\left(-180^{\circ}\right.$ to $180^{\circ}$ ), and the mean of the amplitude of the high-frequency signal $\left(\mathrm{HF}_{\mathrm{amp}}\right)$ occurring within each phase bin was calculated. After applying this procedure on each of the 30 segments, data were then imported back into MATLAB. There, $\mathrm{HF}_{\mathrm{amp}}$ at each phase bin of the low-frequency signal (LF $\phi$ ) was then averaged together across the participant's 30 segments before computing the raw modulation index $\left(\mathrm{MI}_{\text {raw }}\right)$ as the Kullback-Leibler divergence from a uniform amplitude distribution (see Tort et al., 2010 for more details and illustration). To control for factors not of interest that have been shown to affect PAC (such as spectral power), for each participant, 200 surrogate $\mathrm{Ml}$ values $\left(\mathrm{MI}_{\text {surr }}\right)$ were generated by repeating the procedure after offsetting $\mathrm{HF}_{\mathrm{amp}}$ from LF $\phi$ by a randomized time shift between 0.1 and $1.9 \mathrm{~s}$. From this distribution, the mean $\left[\mu\left(\mathrm{MI}_{\text {surr }}\right)\right]$ and SD were then calculated. A normalized $\mathrm{MI}\left(\mathrm{MI}_{\text {norm}}\right)$ was then computed as the $z$ score of the $\mathrm{MI}_{\text {raw }}$ compared with the distribution of $\mathrm{MI}_{\text {surr }}$ values (Canolty et al., 2006). Consequently, for each EEG, a single $\mathrm{MI}_{\text {raw }}, \mu\left(\mathrm{MI}_{\text {surr }}\right)$, and $\mathrm{MI}_{\text {norm }}$ were obtained. For subsequent clustering analysis, $\mathrm{MI}_{\text {raw }}$ values were statistically compared with $\mu\left(\mathrm{MI}_{\text {surr }}\right)$ values, while $\mathrm{MI}_{\text {norm }}$ values were used to assess a correlation with age.

\section{Phase max}

The Tort method used here to quantify PAC captures the presence of coupling, regardless of where in the LF $\phi$ the high-frequency signal demonstrates increased amplitude. However, PAC can result from an increase high-frequency amplitude anywhere in the low-frequency waveform. To capture this, for each frequency pair at each electrode, we determined phase preference by calculating the LF $\phi$ bin where $\mathrm{HF}_{\mathrm{amp}}$ was maximal (termed phase max; Port et al., 2019). Phase max values were averaged across EEG recordings (in each age group, or across all age groups) using the circ_mean function in the circular statistics toolbox (Berens, 2009).

\section{Statistical analysis}

Identification of PAC + clusters across all ages

For every electrode, we now had obtained a modulation index for each frequency pair. To identify statistically significant areas both across frequency pairs and across channels, we employed a cluster-based permutation method described below to identify clusters exhibiting significant PAC (PAC+). We clustered on $\mathrm{MI}$ using data from all useable EEGs, at every age (three months to three years) at which they were collected.

Whereas most clustering procedures involve comparing one condition to another (i.e., task vs baseline stimuli), our task analyzed resting EEG data and therefore had no analogous comparison condition (or baseline) against which to test. In its place, we used the mean modulation index across the surrogate values $\mu\left(\mathrm{MI}_{\text {surr }}\right)$. These data serve as an effective "control" set as it retains the characteristics of the signal (power, noise), while any coupling present in the actual signal should not be retained. Thus, we used clustering against $\mu\left(\mathrm{MI}_{\text {surr }}\right)$ to reveal clusters where the PAC metric of interest is present to a significant degree.

For our clustering procedure, we implemented a nonparametric method that closely followed that by (Maris and Oostenveld, 2007):

1. For every channel, between every filtered low-frequency signal and high-frequency signal, a $t$ test was used to compare the $\mathrm{MI}_{\text {raw }}$ values across all files and the $\mu\left(\mathrm{MI}_{\text {surr }}\right)$ values across all files.

2. Data points where the null hypothesis was rejected ( $p$ $<0.05$, two-sided $t$ test) were selected.

3. Selected data points on the same channel adjacent to one another in terms of low frequency or high frequency were grouped together into clusters (MATLAB function bwlabel, connectivity $=4$ ).

4. After subtracting the minimum $t$ value needed to achieve $p<0.05$ from all data points, cluster level statistics were computed by taking the sum of $t$ values within each cluster.

Then, to compute which clusters were significant (i.e., unlikely to occur with that strength or size by chance, or to remove clusters that may be spurious), we implemented the following:

1. For half of the participants, selected randomly, the $\mathrm{MI}_{\text {raw }}$ and $\mu\left(\mathrm{MI}_{\text {surr }}\right)$ data were "flipped," such that their $\mu\left(\mathrm{MI}_{\text {surr }}\right)$ data were treated as $\mathrm{MI}_{\text {raw }}$, and their $\mathrm{MI}_{\text {raw }}$ were treated as $\mu\left(\mathrm{MI}_{\text {surr }}\right)$.

2. Test statistics and cluster sizes were calculated in the same manner as used previously.

3. The previous steps were repeated 200 times, creating a distribution of cluster sizes.

4 . Clusters $<95$ th percentile of this distribution were removed from further analysis.

For the remaining analysis, only low-frequency, highfrequency, and channel combinations belonging to a cluster found to demonstrate significant PAC $(\mathrm{PAC}+)$ were included.

\section{Development of PAC metrics with age}

To investigate how our PAC metrics in these clusters were associated with age, a Pearson correlation was computed between age and $\mathrm{MI}_{\text {norm }}$ averaged in all analyzed low-frequency, high-frequency, and channel combinations.

To analyze how PAC phase was associated with age, for each EEG recording, the number of low-frequency, high-frequency, and channel combinations analyzed where phase max was found in each LF $\phi$ bin was taken. These values were then divided by the total number of low-frequency, high-frequency, and channel combinations analyzed to obtain the proportion of phase max values found in each phase bin. Age was then correlated with these values in all 18 LF $\phi$ bins, indicating which LF $\phi$ ranges demonstrated increases or decreases in proportion of phase max values with age. Significant correlations $(p<0.05)$ were determined after Bonferroni correction for 18 tests. Because a study being analyzed in parallel observed frontal and posterior channels differed in phase 


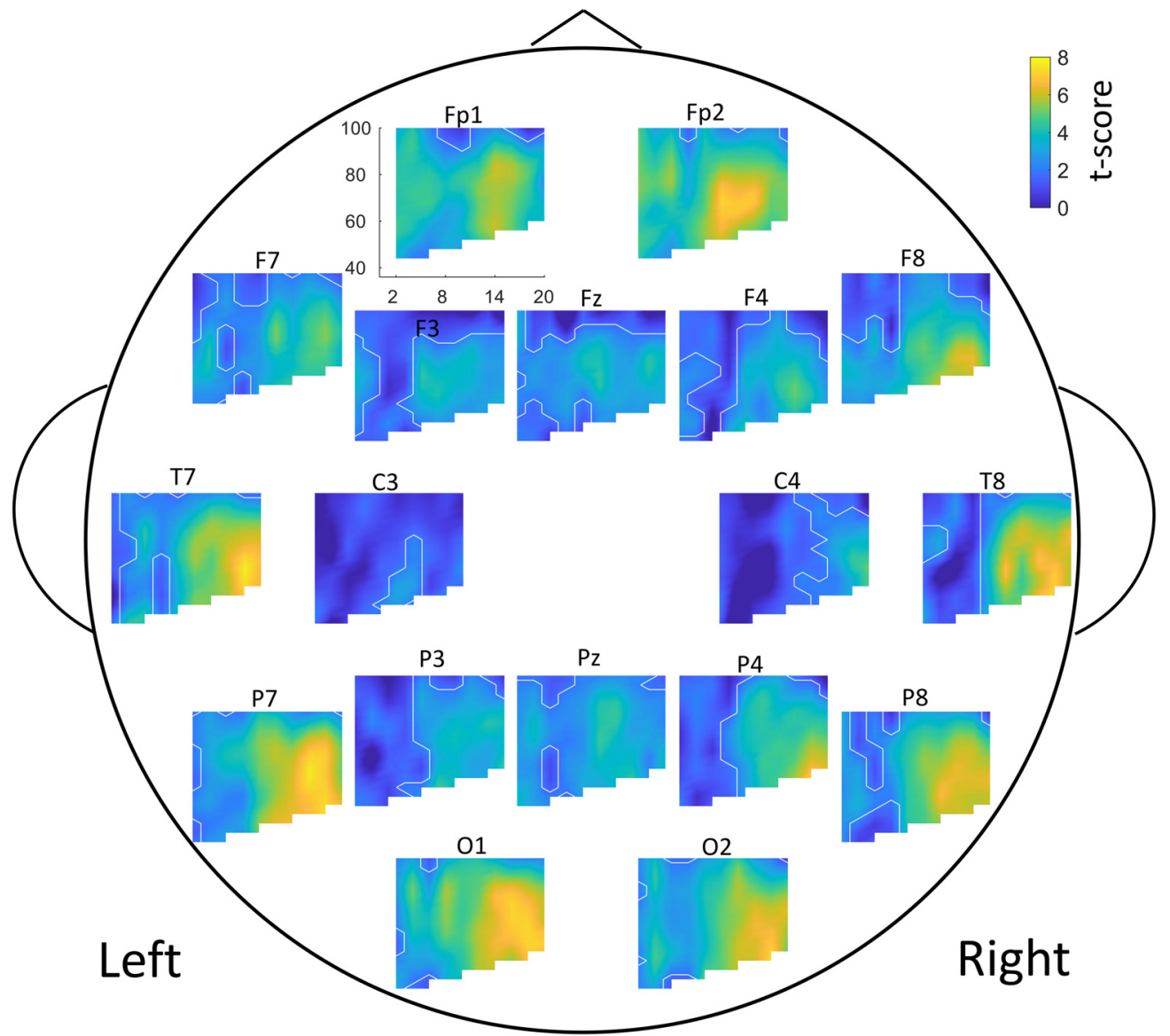

Figure 2. PAC + clusters across all ages. Comodulograms of $t$ scores, for each electrode, showing MI of each area. White lines outline clusters with significant MI (PAC+ clusters, $p<0.05$, corrected for multiple comparisons). In some cases (e.g., Fp1, Fp2, O1, and O2), PAC+ clusters cover most of the comodulogram; therefore, in these cases, white lines outlining blue clusters mark the border of a small area that is not PAC + . Comodulograms indicate the level of coupling between phase frequencies $(x-a x i s, 0-20 \mathrm{~Hz})$, and amplitude frequencies ( $y$-axis, $40-100 \mathrm{~Hz}$ ). Each electrode is plotted at approximate electrode location on scalp. All analyzed EEGs were included (regardless of age at collection).

preference (Mariscal et al., 2021), we additionally performed these correlations in frontal and posterior channels separately.

\section{Code accessibility}

The code to process EEG data are publicly available under the BEAPP and HAPPE software licenses (BEAPP: https://github.com/lcnbeapp/beapp; HAPPE: https://github. com/lcnhappe/happe). Additional code used for calculation of metrics and statistical analysis is available upon reasonable request.

\section{Results}

\section{Simulated signals}

All simulated signals were run through all preprocessing steps (with the exception of re-referencing) and their PAC was subsequently analyzed. All simulated phase max values in each low-frequency phase and high-frequency amplitude combination tested demonstrated its maximum high-frequency amplitude at its approximate simulated phase preference, indicating phase preference was not altered by the filtering steps used during EEG processing or PAC analysis.

\section{Identification of significant MI clusters across all ages}

Clustering on $\mathrm{Ml}$ of all participants regardless of age selected $68.2 \%$ of low-frequency, high-frequency, and channel combinations (Fig. 2). All channels analyzed contained at least one significant cluster.

\section{Phase preference}

There is a spatial distribution of phase max, where more anterior electrodes largely exhibited a phase max on or around $-110^{\circ}$, while more posterior electrodes largely exhibited a phase max on or around $70^{\circ}$ (Figs. 3, 4A).

\section{Development of PAC metrics with age}

$\mathrm{MI}_{\text {norm }}$ in PAC+ clusters increases with age $(r=0.2550$, $\left.p=2.035 \times 10^{-6}\right)$, this increase appears most prominently from one to three years of age (Fig. 5). $\mathrm{MI}_{\text {norm }}$ in PAC+ clusters does not differ as a function of net type $(p>0.1)$. 


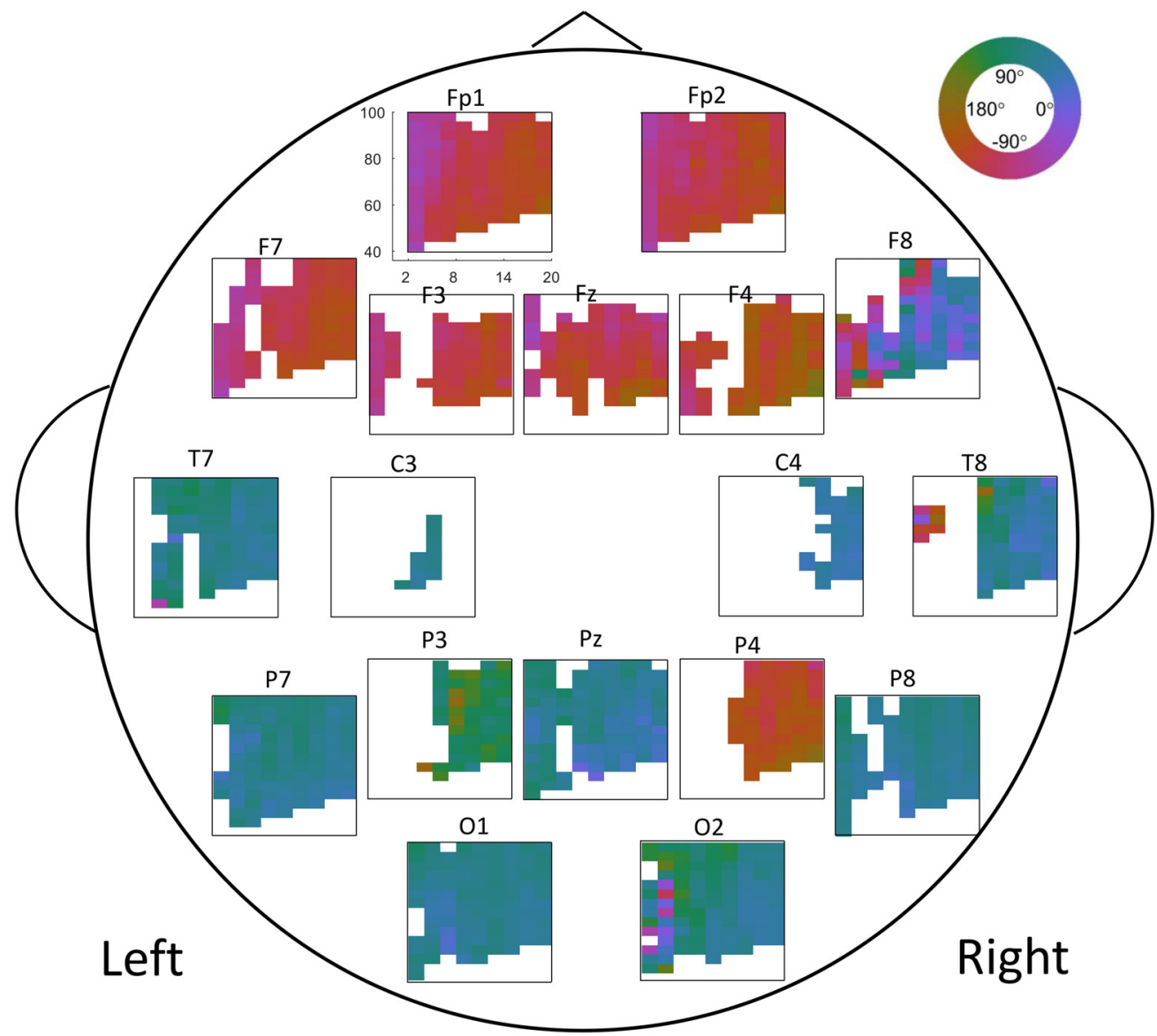

Figure 3. Phase preference across all ages. Comodulograms of phase max, for each electrode. Comodulograms indicate the average phase max value across all EEGs (regardless of age at collection). Here, only low-frequency, high-frequency, and channel combinations in PAC+ clusters are displayed. Each electrode is plotted at approximate electrode location on scalp.

We additionally examined whether the proportion of low-frequency, high-frequency, and channel combinations demonstrating phase max at each phase bin is associated with age (Fig. 4). Over all channels analyzed, the proportion of data points demonstrating phase max in the $90^{\circ}$ phase bin significantly increased with age, while the proportion of data points decreased in the $-30^{\circ}$ phase bin. This change was driven by the posterior channels, where the proportion of data points in the $30^{\circ}-90^{\circ}$ range increased with age, while the proportion of data points in the $-170^{\circ}-\left(-70^{\circ}\right)$ range decreased. Anterior channels, on the other hand, demonstrated a nearly opposite trend, where the proportion of data points in the $-150^{\circ}-\left(-110^{\circ}\right)$ range increased with age, while the proportion of data points in the $-30^{\circ}-70^{\circ}$ phase range, with the exception of the $50^{\circ}$ phase bin, decreased.

\section{Discussion}

The primary goals in this study were to capture the dynamics of early brain development using PAC and to examine how PAC changes over the first three years of life. Here, we explore PAC from three months through three years of age. This PAC occurs most prominently between $\alpha-\beta$ and $\gamma$, largely consistent with several reports of $\alpha-\gamma$ PAC in resting state recordings (Roux et al., 2013; Berman et al., 2015; Gohel et al., 2016) but is also present between theta and $\gamma$. We observe PAC broadly across the scalp, suggesting a relatively ubiquitous presence of cross-frequency coupling. Our data suggest PAC increases in strength with age from three months to three years of age. The phase preference of this PAC shows opposing trends, separated by scalp region: PAC in posterior electrodes (particularly over occipital lobes) is driven by a peak in $\gamma$ amplitude largely during the positive phases of LF $\phi$ (centered around $+70^{\circ}$ ), while PAC in anterior electrodes (particularly over frontal lobes) is driven by a peak in $\gamma$ amplitude largely during the negative phases of the LF $\phi$ [centered around $-130^{\circ}-\left(-110^{\circ}\right)$ ].

To our knowledge, this study is the first to demonstrate this regional separation in phase preference. As a result, the mechanisms underlying this difference, and its relationship to circuit function, is presently unclear. However, the strong difference of phase preference between brain areas, as well as the development of this difference with age, suggests phase preference may capture meaningful network characteristics. For example, laminar recordings have found phase preference to vary by cortical layers (Sotero et al., 2015). Because scalp-level EEG reflects the grand average of underlying activity, one possibility is that 

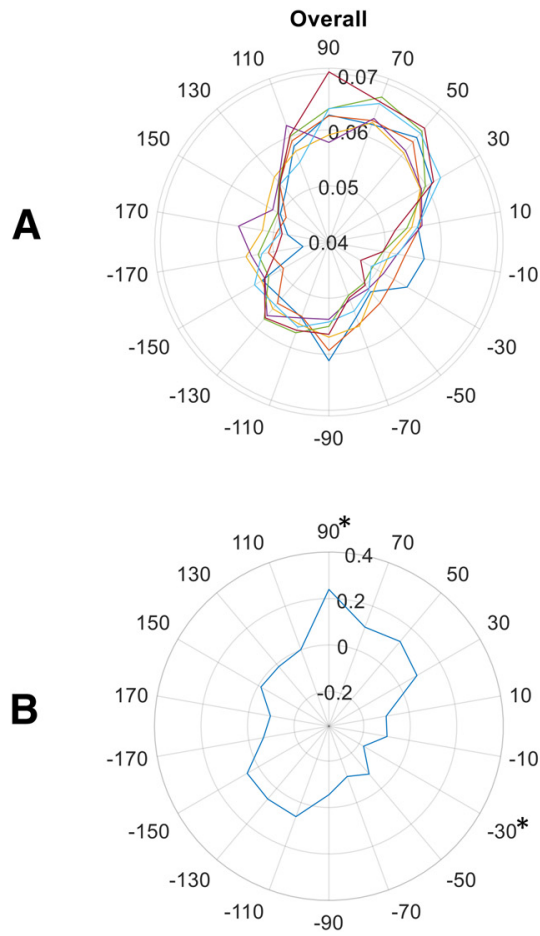
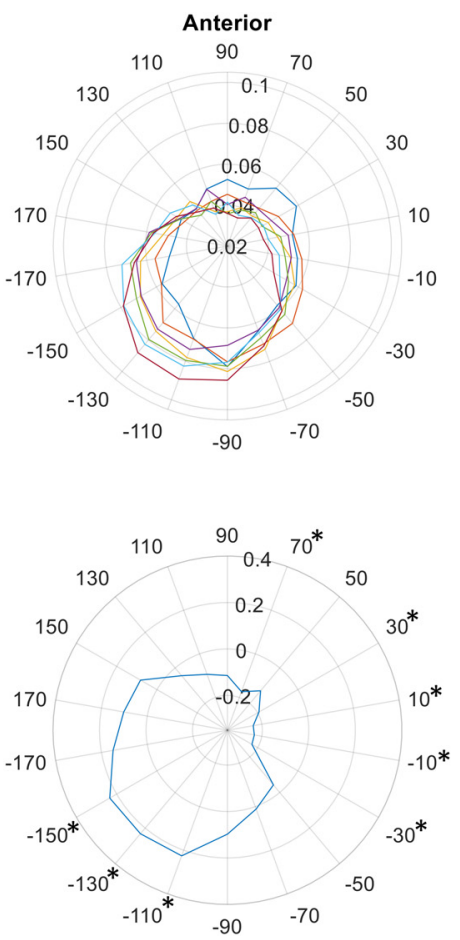
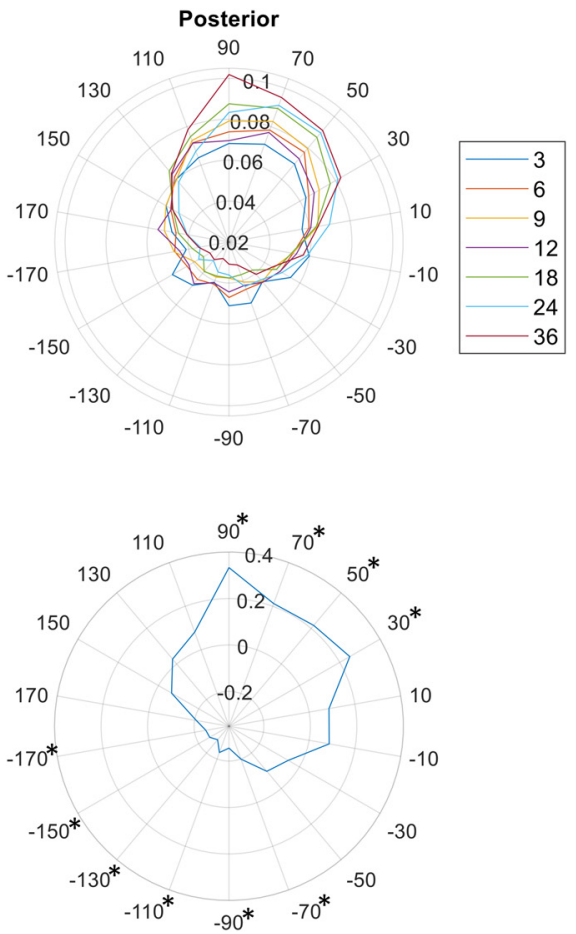

Figure 4. Distribution of phase max across all ages and correlations with age. $\boldsymbol{A}$, Circular plots display the mean proportion of lowfrequency, high-frequency, and electrode combinations demonstrating phase max (radius of figure) at each phase bin (angles of figure) for each age (in months). Data are analyzed and plotted over all channels analyzed (left), anterior channels Fp1, Fp2, F3, F4, F7, F8, Fz (middle), and posterior channels P3, P4, P7, P8, Pz, O1, O2 (right). B, Correlation coefficients ( $\rho$, radius of figure) of the Pearson correlation between the proportion of low-frequency, high-frequency, and electrode combinations demonstrating phase max at each phase bin (angles of figure), and age. *Indicates phase bins where the correlation is significant ( $p<0.05$, Bonferroni corrected for 18 tests).

phase preference reflects the relative activity of underlying cortical layers; this would be of particular mechanistic interest because cortical layers with opposing phase preferences also have opposing predominant directionality of information flow [i.e., feedforward (bottom-up, thalamocortical) vs feedback (top-down, corticothalamic) activity]. Supporting this hypothesis, the relative thickness of each cortical layer differs along the anterior-posterior axis (Wagstyl et al., 2020). Back translation of phase preference findings, perhaps using laminar recordings, may provide further insights into neural network activity across development.

This study is not without limitations. Regarding the dataset analyzed, our sample size at the three-month time point was relatively small $(n=14)$, more participants would allow for a more exact description of PAC at this age. Likewise, most participants did not contribute quality data at every time point, meaning the sample composition at each age differs slightly from other ages. Because of this, our study demonstrates association between age and PAC at a group level, but does not directly demonstrate age-related changes on an individual level. In addition, although PAC has been shown to differ with vigilance state (He et al., 2010; Tokariev et al., 2016), this study only recorded from brief epochs of awake resting data, and consequently these effects were not analyzed. Likewise, our analysis was conducted on only $1 \mathrm{~min}$ of artifact-free data; although analytically, this recording length has been shown to be sufficient for PAC analyses (Berman et al., 2015), a longer recording would provide a more thorough description of PAC for each individual. Finally, the EEG analysis presented here was conducted

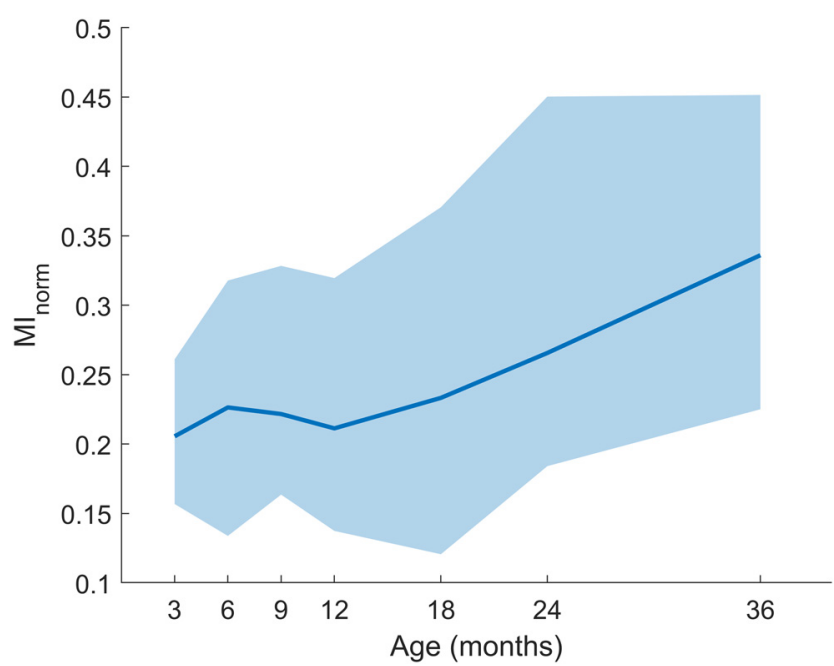

Figure 5. Association between $\mathrm{MI}_{\text {norm }}$ and age. Median $\mathrm{MI}_{\text {norm }}$ in PAC + clusters plotted as a function of age. Clusters between the 25 th and 75 th percentiles are shaded. 
on sensor (not source) level EEG data. This data could therefore be affected by changes in volume conduction because of the structural development of the brain during infancy (Grieve et al., 2003; Odabaee et al., 2014). Future studies are needed to assess how the structural changes in the brain during infancy could affect the results presented here.

In summary, this study documents the emergence of PAC in early childhood. We find $\mathrm{HF}_{\mathrm{amp}}$ is increased at opposing phases of LF $\phi$ in anterior areas as compared with posterior areas. Future studies would be beneficial in further assessing the potential functional relevance of the spatial distribution of phase preference; we suggest laminar differences in the direction of information flow (feedforward vs feedback) as one potential avenue for further exploration.

\section{References}

Berens P (2009) CircStat: a MATLAB toolbox for circular statistics. J Stat Soft 31:1-21.

Berman JI, Liu S, Bloy L, Blaskey L, Roberts TPLL, Edgar JC (2015) Alpha-to-gamma phase-amplitude coupling methods and application to autism spectrum disorder. Brain Connect 5:80-90.

Brito NH, Elliott AJ, Isler JR, Rodriguez C, Friedrich C, Shuffrey LC, Fifer WP (2019) Neonatal EEG linked to individual differences in socioemotional outcomes and autism risk in toddlers. Dev Psychobiol 61:1110-1119.

Canolty RT, Knight RT (2010) The functional role of cross-frequency coupling. Trends Cogn Sci 14:506-515.

Canolty RT, Barbaro NM, Edwards E, Kirsch HE, Dalal SS, Nagarajan SS, Knight RT, Berger MS, Soltani M (2006) High gamma power is phase-locked to theta oscillations in human neocortex. Science 313:1626-1628.

Cho RY, Walker CP, Polizzotto NR, Wozny TA, Fissell C, Chen CMA, Lewis DA (2015) Development of sensory gamma oscillations and cross-frequency coupling from childhood to early adulthood. Cereb Cortex 25:1509-1518.

Delorme A, Makeig S (2004) EEGLAB: an open source toolbox for analysis of single-trial EEG dynamics including independent component analysis. J Neurosci Methods 134:9-21.

Dupré la Tour T, Tallot L, Grabot L, Doyère V, van Wassenhove V, Grenier Y, Gramfort A (2017) Non-linear auto-regressive models for cross-frequency coupling in neural time series. PLoS Comput Biol 13:e1005893.

Gabard-Durnam LJ, Mendez Leal AS, Wilkinson CL, Levin AR (2018) The Harvard automated processing pipeline for electroencephalography (HAPPE): standardized processing software for developmental and high-artifact data. Front Neurosci 12:97.

Gabard-Durnam LJ, Wilkinson C, Kapur K, Tager-Flusberg H, Levin AR, Nelson CA (2019) Longitudinal EEG power in the first postnatal year differentiates autism outcomes. Nat Commun 10:4188.

Gao W, Lin W, Grewen K, Gilmore JH (2017) Functional connectivity of the infant human brain. Neuroscientist 23:169-184.

Gohel B, Lim S, Kim MY, An KM, Kim JE, Kwon H, Kim K (2016) Evaluation of phase-amplitude coupling in resting state magnetoencephalographic signals: effect of surrogates and evaluation approach. Front Comput Neurosci 10:120.

Grieve PG, Emerson RG, Fifer WP, Isler JR, Stark RI (2003) Spatial correlation of the infant and adult electroencephalogram. Clin Neurophysiol 114:1594-1608.

He BJ, Zempel JM, Snyder AZ, Raichle ME (2010) The temporal structures and functional significance of scale-free brain activity. Neuron 66:353-369.

Helfrich RF, Herrmann CS, Engel AK, Schneider TR (2016) Different coupling modes mediate cortical cross-frequency interactions. Neuroimage 140:76-82.
Hyafil A, Giraud A-L, Fontolan L, Gutkin B (2015) Neural cross-frequency coupling: connecting architectures, mechanisms, and functions. Trends Neurosci 38:725-740.

Jensen O, Gips B, Bergmann TO, Bonnefond M (2014) Temporal coding organized by coupled alpha and gamma oscillations prioritize visual processing. Trends Neurosci 37:357-369.

Jones KT, Johnson EL, Berryhill ME (2020) Frontoparietal thetagamma interactions track working memory enhancement with training and tDCS. Neuroimage 211:116615.

Kopell NJ, Gritton HJ, Whittington MA, Kramer MA (2014) Beyond the connectome: the dynome. Neuron 83:1319-1328.

Lega B, Burke J, Jacobs J, Kahana MJ (2016) Slow-theta-togamma phase-amplitude coupling in human hippocampus supports the formation of new episodic memories. Cereb Cortex 26:268-278.

Levin AR, Méndez Leal AS, Gabard-Durnam LJ, O’Leary HM (2018) BEAPP: the batch electroencephalography automated processing platform. Front Neurosci 12:513.

Maris E, Oostenveld R (2007) Nonparametric statistical testing of EEG- and MEG-data. J Neurosci Methods 164:177-190.

Mariscal M, Berry-Kravis E, Buxbaum J, Ethridge L, Filip-Dhima R, Foss-Feig J, Kolevzon A, Modi M, Mosconi M, Nelson C, Powell C, Siper P, Soorya L, Thaliath A, Thurm A, Zhang B, Sahin M, Levin A; Developmental Synaptopathies Consortium (2021) Shifted phase of EEG cross-frequency coupling in individuals with PhelanMcDermid syndrome. Mol Autism 12:29.

Mullen T (2012) CleanLine EEGLAB Plugin. Neuroimaging informatics tools and resources clearinghouse (NITRC). Available from https://www.nitrc.org/projects/cleanline/.

Ninomiya T, Dougherty K, Godlove DC, Schall JD, Maier A (2015) Microcircuitry of agranular frontal cortex: contrasting laminar connectivity between occipital and frontal areas. J Neurophysiol 113:3242-3255.

Odabaee M, Tokariev A, Layeghy S, Mesbah M, Colditz PB, Ramon C, Vanhatalo S (2014) Neonatal EEG at scalp is focal and implies high skull conductivity in realistic neonatal head models. Neuroimage 96:73-80.

Onton J, Makeig S (2006) Information-based modeling of event-related brain dynamics. Prog Brain Res 15:999-120.

Port RG, Berman JI, Liu S, Featherstone RE, Roberts TPL, Siegel SJ (2019) Parvalbumin cell ablation of NMDA-R1 leads to altered phase, but not amplitude, of gamma-band cross-frequency coupling. Brain Connect 9:263-272.

Roux F, Wibral M, Singer W, Aru J, Uhlhaas PJ (2013) The phase of thalamic alpha activity modulates cortical gamma-band activity: evidence from resting-state MEG recordings. J Neurosci 33:17827-17835.

Saby JN, Marshall PJ (2012) The utility of EEG band power analysis in the study of infancy and early childhood. Dev Neuropsychol 37:253-273.

Sahin M, Jones SR, Sweeney JA, Berry-Kravis E, Connors BW, Ewen JB, Hartman AL, Levin AR, Potter WZ, Mamounas LA (2019) Discovering translational biomarkers in neurodevelopmental disorders. Nat Rev Drug Discov 18:235-236.

Salimpour Y, Anderson WS (2019) Cross-frequency coupling based neuromodulation for treating neurological disorders. Front Neurosci 13:125.

Soplata AE, McCarthy MM, Sherfey J, Lee S, Purdon PL, Brown EN, Kopell N (2017) Thalamocortical control of propofol phase-amplitude coupling. PLoS Comput Biol 13:e1005879.

Sotero RC, Bortel A, Naaman S, Mocanu VM, Kropf P, Villeneuve M, Shmuel A (2015) Laminar distribution of phase-amplitude coupling of spontaneous current sources and sinks. Front Neurosci 9:454.

Tokariev A, Videman M, Palva JM, Vanhatalo S (2016) Functional brain connectivity develops rapidly around term age and changes between vigilance states in the human newborn. Cereb Cortex 26:4540-4550. 
Tort ABL, Komorowski R, Eichenbaum H, Kopell N (2010) Measuring phase-amplitude coupling between neuronal oscillations of different frequencies. J Neurophysiol 104:1195-1210.

Vanhatalo S, Matias Palva J, Andersson S, Rivera C, Voipio J, Kaila K (2005) Slow endogenous activity transients and developmental expression of $\mathrm{K}+-\mathrm{Cl}$ - cotransporter 2 in the immature human cortex. Eur J Neurosci 22:2799-2804.

Voytek B (2010) Shifts in gamma phase-amplitude coupling frequency from theta to alpha over posterior cortex during visual tasks. Front Hum Neurosci 4:191.

Wagstyl K, Larocque S, Cucurull G, Lepage C, Cohen JP, Bludau S, Palomero-Gallagher N, Lewis LB, Funck T, Spitzer H, Dickscheid T, Fletcher PC, Romero A, Zilles K, Amunts K, Bengio Y, Evans AC
(2020) BigBrain 3D atlas of cortical layers: cortical and laminar thickness gradients diverge in sensory and motor cortices. PLOS Biol 18:e3000678.

Winkler I, Haufe S, Tangermann M (2011) Automatic classification of artifactual ICA-components for artifact removal in EEG signals. Behav Brain Funct 7:30.

Winkler I, Brandl S, Horn F, Waldburger E, Allefeld C, Tangermann M (2014) Robust artifactual independent component classification for BCl practitioners. J Neural Eng 11:035013.

Wolff JJ, Piven J (2020) Predicting autism risk in infancy. J Am Acad Child Adolesc Psychiatry, in press.

Yael D, Vecht JJ, Bar-Gad I (2018) Filter-based phase shifts distort neuronal timing information. eNeuro 5:ENEURO.0261-17.2018. 